\title{
Exploring dual-frequency sub-Doppler spectroscopy for a Cs microcell-based optical frequency reference
}

\author{
M. Petersen ${ }^{1}$, G. Coget $^{1}$, V. Maurice ${ }^{1,3}$, C. Gorecki ${ }^{1}$, N. Passilly ${ }^{1}$, D. Brazhnikov ${ }^{2}$, R. Boudot ${ }^{1,3}$ \\ (1) - FEMTO-ST, CNRS, UBFC, 26 chemin de l'Epitaphe, 25030 Besançon, France \\ (2) - Institute of Laser Physics, SB RAS Novosibirsk State University Novosibirsk, Russia \\ (3) - now at National Institute of Standards and Technology, Boulder, Colorado, 80309, USA \\ Email: rodolphe.boudot@femto-st.fr
}

\begin{abstract}
Dual-frequency sub-Doppler spectroscopy (DF SDS) in vapor cells allows the detection of enhanced-absorption natural-line-width resonances. We investigate here the application of this spectroscopy technique into a Cs micro-fabricated cell. The dependence of the sub-Doppler resonance on the laser intensity is described. The influence of the cell temperature and the retroreflection mirror position was also studied. A preliminary laser beat-note between two $894 \mathrm{~nm}$ lasers, each stabilized using DF SDS, demonstrates an Allan deviation lower than $2 \times 10^{-12}$ at $1 \mathrm{~s}$ and at the level of $10^{-12}$ at $10^{2} \mathrm{~s}$. Main contributions to the short-term and mid-term stability budget are under study. Latest results will be reported at the conference.
\end{abstract}

\section{INTRODUCTION}

Inspired by significant progress demonstrated in the domain of state-of-the-art optical clocks [1], [2] and MEMS cellbased atomic frequency references [3], [4], [5], an exciting research investigation path is to propose the development of next-generation ultra-miniaturized cell-based optical frequency references. In this field, original studies and results have already been reported. This includes the study of Dopplerfree resonances in microfabricated cells [6], the stabilization of VCSELs onto Doppler-broadened resonances [7], the stabilization of a Brillouin scattering laser onto a $\mathrm{Rb}$ microcell [8], sub-Doppler spectroscopy technique using an integrated photonic waveguide [9] or the recent photonic integration of a miniaturized optical clock based on detection of the Rb $778 \mathrm{~nm}$ two-photon transition in a microfabricated cell [10]. The latter frequency reference exhibits a remarkable frequency instability level of $4.4 \times 10^{-12} \tau^{-1 / 2}$ until $1000 \mathrm{~s}$ [10] whereas previous works [7], [8], [9] were often limited at the level of about $10^{-11}$ between $1 \mathrm{~s}$ and $10^{4} \mathrm{~s}$. Note also excellent frequency noise performances reported by ultralow noise miniaturized external cavity semiconductor laser using whispering-gallery mode optical micro-resonators [11].

In a recent study [12], the detection of high-contrast signreversed natural-linewidth Doppler-free dips in a Cs vapor cell has been reported using dual-frequency sub-Doppler spectroscopy (DF SDS). Properties of these enhanced-absorption spikes, explained by the contribution of Zeeman dark states, hyperfine dark states and optical pumping effects, has been investigated in [13]. This technique has been employed to improve the frequency stability of Cs vapor cell atomic clocks [14], [15]. More recently, an extended theoretical model of DF SDS has been developed [16], highlighting the interest to apply this spectroscopy technique to short-length cells since optimized contrast of the sub-Doppler resonance can be obtained with proper adjustment of the retroreflection mirror. In the present work, we propose to investigate the potential of DF SDS in a Cs microfabricated vapor cell for laser frequency stabilization application. We describe the experimental setup. We report preliminary Allan deviation results of a laser beatnote between two lasers (one ECDL and one DFB), each frequency-stabilized using the DF SDS technique.

\section{EXPERIMENTAL SETUP}

The experimental setup is analog to a standard saturated absorption scheme setup [17], [18]. The main difference is that, instead of being illuminated by two counter-propagating single-frequency optical fields, atoms in the cell interact with two counter-propagating dual-frequency optical fields [12]. Both counter-propagating beams have orthogonal linear polarizations [12], [13]. The frequency difference between both optical lines of the bi-chromatic field is close to the hyperfine splitting frequency $(9.192 \mathrm{GHz}$ for $\mathrm{Cs}$ atom).

The laser source is an extended cavity diode laser, tuned on the Cs $D_{1}$ line at $895 \mathrm{~nm}$. An optical isolator is placed at the output of the laser to prevent optical feedback. The laser beam, with a diameter of $1 \mathrm{~mm}$, is directed into a Cs vapor micro-fabricated cell. The cell technology is analog to the one described in [19], without buffer gas. The science cavity of the cell is $2 \mathrm{~mm}$ diameter and 1.4-mm long. The cell is temperature stabilized and surrounded by a mu-metal magnetic shield. At the output of the cell, the dual-frequency laser beam is reflected by a mirror. The laser beam crosses the cell a second time and is detected by a photodiode at the output of the cell.

\section{EXPERIMENTAL RESULTS}

We measured the line-width and height of the sub-Doppler resonance for a cell temperature of $60^{\circ} \mathrm{C}$. We found that the linewidth gets close to the natural-linewidth for small laser power values and is increased with increased laser power, yielding $50 \mathrm{MHz}$ for a laser power of $1 \mathrm{~mW}$. The height of the resonance is increased with the laser power up to about $200 \mu \mathrm{W}$, before reaching a saturation plateau.

We stabilized the frequency of the ECDL onto the sub-Doppler resonance detected in the $\mathrm{Cs}$ vapor microcell. For this purpose, the laser current is slightly modulated (to modulate the laser frequency). The voltage signal at the output of the photodiode is synchronously demodulated with a dedicated digital lock-in amplifier. The zero-crossing error signal is then processed into 
a PI controller and used to correct the laser frequency. The ECDL output beam is superimposed with another laser beam, coming from a distributed-feedback (DFB) laser, stabilized using DF SDS onto a 2-cm and 2-cm diameter long Cs vapor cell. Using a fast photodiode, a laser beat-note between both lasers at about $4.6 \mathrm{GHz}$ is generated. This signal is mixed with the microwave signal coming from a microwave frequency synthesizer, driven by a hydrogen maser. A final beat-note at about $22 \mathrm{MHz}$ is then counted with a frequency counter.

The Allan deviation of the laser beat-note is measured, in a preliminary way, at the level of $2 \times 10^{-12}$ at $1 \mathrm{~s}$ and $10^{-12}$ at $100 \mathrm{~s}$ integration time. This preliminary short-term stability result at $1 \mathrm{~s}$ is encouraging and is about 50-100 times better at $1 \mathrm{~s}$ than commercial chip-scale atomic clocks [20]. This demonstrates that DF SDS is an interesting approach for the development of a cell-based optical frequency reference, with potential for miniaturization. Studies are in progress to estimate main contributions to the optical frequency reference fractional frequency stability at $1 \mathrm{~s}$ and $100 \mathrm{~s}$. Latest results will be presented at the conference.

\section{ACKNOWLEDGMENT}

The authors thank P. Abbé, R. Vicarini and C. Rocher (FEMTO-ST) for their help with experimental stuff. This work was supported by Région Bourgogne Franche-Comté. D. Brazhnikov thanks Russian Science Foundation (17-7220089). The authors thank LabeX FIRST-TF and the EquiPX Oscillator-IMP platform.

\section{REFERENCES}

[1] M. Schioppo et al, "Ultrastable optical clock with two cold-atom ensemble" Nature Photonics 11, 48-52 (2017)

[2] [2] N. Huntemann et al. "Single-ion atomic clock with $310^{-18}$ systematic uncertainty", Phys. Rev. Lett. 116, 063001 (2016).

[3] S. Knappe et al. "A microfabricated atomic clock", Appl. Phys. Lett. 85, 9, 1460 (2004).

[4] R. Lutwak, et al., "The Chip-Scale atomic clock - recent developments", in Proc. 2009 Joint Meeting IEEE Int. Freqency Control Symp. and EFTF Conf., Besancon, France, 573-577 (2009).

[5] J. E. Kitching "Chip-scale atomic devices", App. Phys. Rev. 5031302 (2018).

[6] S. Knappe, H. G. Robinson and L. Hollberg, "Microfabricated saturated absorption laser spectrometer" Opt. Express 15, 10, 6293-6298 (2007).

[7] F. Gruet et al., Opt. Lasers Eng 51, 8, 1023-1027 (2013).

[8] [8] W. Loh, M. T. Hummon, H. F. Leopardi, T. M. Fortier, F. Quinlan, J. Kitching, S. B. Papp and S. A. Diddams, "Microresonator Brillouin laser stabilization using a microfabricated rubidium cell", Opt. Express 24, 13, 14516-14524 (2016).

[9] M. T. Hummon, S. Kang, D. G. Bopp, Q. Li, D. A. Westly, S. Kim, C. Fredrick, S. A. Diddams, K. Srinivasan, V. Aksyuk and J. Kitching, "Photonic chip for laser stabilization to an atomic vapor with $10^{-11}$ instability", Optica 5, 4, 443-449 (2018).

[10] Z. L. Newmann et al, "Photonic integration of an optical atomic clock", ArxiV 1811.00616 (2018).

[11] W. Liang et al., "Ultralow noise miniature external cavity semiconductor laser", Nature Comm. 8371, 1-6 (2015).

[12] M. Abdel Hafiz, G. Coget, E. de Clercq, and R. Boudot, "Doppler-free spectroscopy on Cs D1 line with a dual-frequency laser", Opt. Lett. 41, 2982 (2016).

[13] M. Abdel Hafiz, D. Brazhnikov, A. Taichenachev, V. Yudin, E. de Clercq and R. Boudot, "High-contrat sub-Doppler absorption spikes in a hot atomic vapor cell exposed to a dual-frequency laser field", New J. Phys. 19, 073028 (2017).
[14] M. Abdel Hafiz, G. Coget, P. Yun, S. Guerandel, E. de Clercq and R. Boudot, "A high-performance Raman-Ramsey Cs vapor cell atomic clock", J. Appl. Phys. 121, 104903 (2017).

[15] P. Yun, F. Tricot, C. E. Calosso, S. Micalizio, B. Francois, R. Boudot, S. Guerandel and E. de Clercq, "High-performance coherent-population trapping clock with polarization modulation", Phys. Rev. Applied 7, 014018 (2017).

[16] D. Brazhnikov, M. Petersen, G. Coget, V. Maurice, N. Passilly, C. Gorecki and R. Boudot, "Dual-frequency sub-Doppler spectroscopy: Extended theoreticql model and microcell-based experiments", ArxiV 1812.09641 (2018).

[17] V. S. Letokhov and V. P. Chebotaev, "Nonlinear laser spectroscopy", Springer, Berlin (1977).

[18] W. Demtroder, "Laser spectroscopy. Basic concepts and insrumentation", Third edition, Springer-Verlag, Berlin (2003).

[19] R. Vicarini, V. Maurice, M. Abdel Hafiz, J. Rutkowski, C. Gorecki, N. Passilly, L. Ribetto, V. Gaff, V. Volant, S. Galliou, and R. Boudot,"Demonstration of the mass-producible feature of a Cs vapor microcell technology for miniature atomic clocks" Sens. Actuators A: Phys. 280, 99-106 (2018).

[20] https://www.microsemi.com/product-directory/clocks-frequencyreferences/3824-chip-scale-atomic-clock-csac 